\title{
Celiac artery compression syndrome. Mini-review
}

\author{
Magdalena Rongies-Kosmol, Tomasz Jakimowicz \\ Department of General, Vascular and Transplant Surgery, The Medical University of Warsaw, Poland
}

\begin{abstract}
Celiac artery compression syndrome is a rare vascular disease, with incidence estimated at $0.4 \%$. However, asymptomatic but hemodynamically significant celiac artery compression is found incidentally in $2.4-8 \%$ of the population on examination. The disease is caused by compression of the median arcuate ligament and celiac axis nerve fibers on the celiac artery, usually during expiration, and can cause symptoms of "abdominal claudication". These symptoms include post-prandial epigastric pain, nausea, vomiting, mild weight loss and, less frequently, diarrhea, heartburn, abdominal bloating, constipation, arrhythmias and syncope. Ultrasound, computed tomography (CT), magnetic resonance (MR) and angiography are employed to diagnose celiac artery compression syndrome. Treatment of this disease is based on median arcuate ligament lysis and celiac ganglionectomy either by laparoscopic or open method, with both techniques having similar outcomes.
\end{abstract}

Key words: celiac artery compression syndrome, celiac trunk, median arcuate ligament, celiac plexus

Acta Angiol 2015; 21 , I: $21-24$

\section{Introduction}

Celiac artery compression syndrome (CACS) is a rare disease occurring in $0.4 \%$ of the population [I], although incidental finding of hemodynamically significant yet asymptomatic celiac artery compression occurs in $2.4-8 \%$ of the population [ $[-3]$. The disease is caused by overgrowth of inferiorly placed median arcuate ligament of the diaphragm, causing celiac artery compression during the expiration phase. It can cause symptoms of "abdominal claudication" [4, 5]. An intrinsic component of the disease's pathogenesis is the irritation of the celiac plexus fibers by the overgrown ligament. Common symptoms include post-prandial epigastric pain, nausea, vomiting, and mild weight loss. Less frequently patients experience diarrhea, heartburn, abdominal bloating, constipation, arrhythmias and syncope [6-9].
The anatomical description of celiac artery compression was first published by Lipshutz in 1917 [10]. Celiac artery compression syndrome was later identified by Harjola in 1963 [II] and Dunbar in 1965 [12].

In the contemporary literature, singular cases of diagnosis and treatment of the disease can be found. There are hospitals where, up until 2013 , more than 30 patients diagnosed with the syndrome were operated. In the case of pediatric patients under 21 years, University of Chicago Medicine is the leading institution, where 46 patients underwent laparoscopic surgery for the condition between 2008 and 2012 [13]. In University Hospital Dusseldorf, 18 patients with CACS were operated with open method from 2000 to 2009 [7]. In turn, 5I CACS-diagnosed patients were operated in San Francisco hospital until 1984 [14]. Singular cases of typical diagnosis and treatment of CACS can be found in medical literature from 2013, e.g. a paper by Sang Jin Kim from Korea [15].

Address for correspondence: Tomasz Jakimowicz, e-mail: tomj@amwaw.edu.pl, Magdalena Rongies-Kosmol, The Medical University of Warsaw, Banacha IA, 02-097 Warsaw, tel.: +48225992467 


\section{Anatomy}

The celiac trunk is a single celiac branch of the abdominal aorta. It usually originates between the levels of $12^{\text {th }}$ thoracic and first lumbar vertebrae. The end of the celiac artery lies above the upper edge of pancreas and is 15-20 mm long. Typically, it then divides into three arteries: left gastric artery, splenic artery and hepatic artery, which supply stomach, spleen, pancreas, liver, and duodenum [16].

The diaphragm is divided into lumbar, costal and sternal parts. CACS pathogenesis is related to the lumbar part which originates bilaterally with three arcuate ligaments (median, medial, lateral) and diaphragmatic crura. Median arcuate ligament is a fibrous arch that unites the diaphragmatic crura crossing aorta superior to the celiac axis at the level of the first lumbar vertebra. In addition, another formation surrounding and impinging on the celiac artery is the celiac plexus which comprises of celiac nerves and branches of vagus and phrenic nerves. They are responsible for stimulating the muscular membrane of sphincter muscles in the digestive tract, halting the activity of the other digestive tract muscles, conducting pain stimuli from abdominal organs, and causing abdominal artery stenosis [8, 16].

\section{Diagnosis}

The following three conditions need to be satisfied in order to diagnose CACS:

- occurrence of characteristic symptoms of the syndrome;

- exclusion of other causes of the symptoms (ulcerative disease, cancer, etc.);

- confirmation of celiac trunk stenosis without atherosclerosis through additional examination (duplex ultrasound, computer tomography [CT], magnetic resonance [MR], angiography).

Currently, in order to confirm CACS contrast enhanced CT is the gold standard. Other examinations leading to diagnosis are: MR, Doppler ultrasound evaluating blood flow velocity in the celiac artery, and arteriography which is becoming less popular due to its invasive nature. Each of these examinations should be performed both during inspiration and expiration, as the largest compression of the median arcuate ligament occurs during deep expiration but is not always visible during the inspiration phase [17].

Characteristic features on CT angiogram include celiac artery stenosis, significant hooked appearance of the artery in 3D reconstruction in sagittal plane, and post-stenotic dilatation of proximal celiac artery [4].

On ultrasound Doppler, significant stenosis of celiac arteries is thought to occur when the velocity of the blood flow increases two-fold in comparison to the velocity of the blood flow in aorta. It is commonly agreed that the above-mentioned examination is the most accurate form of screening for people with suspected CACS. This is due to the fact that it is a non-invasive, relatively inexpensive examination without adverse effects such as radiation. In addition, ultrasound Doppler is a very effective tool for post-operative control of patients with CACS. The most objective and comparable indicator is the evaluation of velocity of the blood flow in celiac artery before and after surgery. In a study of 46 pediatric patients under 21 years, preoperative mean peak systolic velocity (PSV) amounted to $38 \mathrm{I} \mathrm{cm} / \mathrm{s}$, whereas postoperative PSV equaled $235 \mathrm{~cm} / \mathrm{s}$. This decrease in pressure corresponded to reduced compression of celiac artery and relief of symptoms in majority of cases [13].

\section{Epidemiology}

CACS occurs in $0.4 \%$ of the population. However, one has to bear in mind that symptom-free existence of celiac trunk compression is estimated at $2.4-8 \%$ [1-3]. Authors from Korea prospectively evaluated the incidence and etiologies of celiac axis stenosis in 400 arteriograms of patients referred to them for chemoembolization of hepatic tumors. All patients were free from symptoms of CACS. It turned out that $7.3 \%$ patients had celiac axis stenosis, in $55 \%$ the etiology was extrinsic compression by the median arcuate ligament [2]. Another study confirming symptom-free occurrence of CACS is a retrospective analysis of 744 patients who underwent CT of the abdominal aorta for various medical indications. 2 I patients $(2.8 \%)$ were shown to have compression of the celiac artery by the ligament, but only 3 of them had symptoms of CACS such as postprandial epigastric pain, vomiting or diarrhea [I]. Due to the frequent incidental finding of asymptomatic celiac artery stenosis on imaging studies, treatment should be dedicated to patients with symptomatic CACS only.

CACS concerns mainly young people between 30 and 50 years of age, in particular women (75-91\%) $[7,13,18]$. There are also reports of familial CACS. In 2010, the case of a 16-year-old girl operated for CACS whose mother had previously been treated for the same disease was reported [19]. In 20II, the case of $6 \mathrm{I}$-year-old man with hepatitis B-related cirrhosis and hepatocellular carcinoma awaiting transplantation was described. After computed tomography, celiac artery stenosis was detected. The patient, however, was free from CACS symptoms. His four children also underwent a CT in order to qualify as candidates to be living related liver donors. Each of the children was diagnosed 
with celiac artery compression by the median arcuate ligament. None of them had any digestive problems or abdominal pains. According to the authors of the study, this is an unequivocal proof of a familial pattern in CACS [20].

It has to be mentioned that the diagnostic process of CACS is usually long, as most patients are qualified for operative treatment after months, and sometimes even years, of gastroenterological examinations, imaging, and sometimes psychiatric consultations. It seems that the main reason behind the status lies with unspecific symptoms but also doctors' lack of awareness about CACS.

Until present, there have been no risk factors identified predisposing for the disease.

\section{Treatment}

In CACS, as the name indicates, compression is the source of pathology and has to be addressed surgically. Preferred treatment involves cutting median arcuate ligament and celiac ganglionectomy which can be performed either by laparoscopic or open method. Both procedures are safe and effective. No death has been reported as a postoperative complication and the percentage of patients with immediate symptomatic relief is $85 \%$ [17].

However, even in treated cases, symptoms can persist or recur. In cases of incomplete resolution of symptoms, endovascular treatment can be employed as a further therapeutic method. After re-diagnosis and visualization of celiac artery stenosis, endovascular treatment based on celiac artery PTA with possible stent implantation should be considered. The abovementioned practice was successful in two reported cases and in the follow-up period of 6 and 15 months, symptoms have not reappeared [5, 2I].

Medical literature also mentions cases where primary endovascular treatment failed and required serious reconstructive surgery. The case of a $2 \mathrm{I}$-year-old woman with CACS who had celiac artery PTA and stenting has been reported. Two months after the procedure symptoms recurred as a result of restenosis. PTA and stenting were repeated. Unfortunately, after 6 weeks early relapse of the condition was diagnosed on grounds of celiac artery stenosis between the two previous stents. The patient was moved to another treatment center where PTA and stenting were performed for the third time. This time the intervention also did not bring satisfying results. In the end the patient was qualified for an open surgery. During the operation damage of stents was established and was followed by a reconstruction of celiac artery with a side-to-end aortoceliac 8-mm Hemashield Dacron interposition graft. Only after this form of treatment satisfying result was obtained and at a 28-month follow-up the symptoms have not recurred. Description of this particular case indicates that endovascular treatment of CACS should not be the preferred method of treatment [6].

\section{Conclusions}

Celiac artery compression syndrome is a rarely diagnosed disease with nonspecific clinical symptoms, and one that should be considered after exclusion of other causes of abdominal symptoms. Preferred treatment method of CACS is median arcuate ligament lysis and celiac ganglionectomy either by laparoscopic or open surgery. Results of both methods are comparable, with a high ratio of cured patients and small number of complications.

\section{References}

I. Gümüş H, Gümüş M, Tekbaş $G$ et al (20/2) Clinical and multidetector computed tomography findings of patients with median arcuate ligament syndrome. Clinical Imaging; 36: 522-525.

2. Park CM, Chung JW, Kim HB, Shin SJ, Park JH (200I) Celiac axis stenosis: incidence and etiologies in asymptomatic individuals. Korean J Radiol; 2: 8-13.

3. Soman S, Sudhakar SV, Keshava SN (2010) Celiac axis compression by median arcuate ligament on computer tomography among asymptomatic person. Indian J Gastroenterol; 29: $121-123$.

4. Horton KM, Talamini MA, Fishman EK (2005) Median arcuate ligament syndrome: evaluation with $C T$ angiography. RadioGraphics; 25: II77-1 I82.

5. Hongsakul K, Rookkapan S, Sungsiri J, Tubtawee T (2012) A severe case of median arcuate ligament syndrome with successful angioplasty and stenting. Hindawi Publishing Corporation Case Reports in Vascular Medicine; Article ID I29870.

6. Delis KT, Gloviczki P, Altuwaijri M, McKusick MA (2007) Median arcuate ligament syndrome: Open celiac artery reconstruction and ligament division after endovascular failure. J Vasc Surg; 46: 799-802.

7. Grotemeyer D, Duran M, Iskandar F, Blondin D, Nguyen K, Sandmann W (2009) Median arcuate ligament syndrome: vascular surgical therapy and follow-up of 18 patients. Langenbecks Arch Surg; 394: 1085-1092.

8. Vaziri K, Hungness ES, Pearson EG, Soper NJ (2009) Laparoscopic treatment of celiac artery compression syndrome: case series and review of current treatment modalities. J Gastrointest Surg; 13: 293-298.

9. Schweizer P, Berger S, Schweizer M, Schaefer J, Beck O (2005) Arcuate ligament vascular compression syndrome in infants and children. J Pediatric Surg; 40: 1616-1622.

10. Lipshutz B (1917) A composite study of the coeliac axis artery. Ann Surg; 65: 159.

11. Harjola PT (1963) A rare obstruction of the coeliac artery. Report of a case. Ann Chir Gynaecol Fenn; 52: 547-550.

12. Dunbar JD, Molnar W, Beman FF (1965) Compression of the celiac trunk and abdominal angina. Am J Roentgenol Radium Ther Nucl Med; 95: 73I-744. 
13. Mak GZ, Speaker Ch, Anderson K et al (2013) Median arcuate ligament syndrome in pediatric population. J Pediatr Surg; 48: 2261-2270.

14. Reilly LM, Ammar AD, Stoney RJ, Ehrenfeld WK (1985) Late results following operative repair for celiac artery compression syndrome. J Vasc Surg; 2: 79-91.

15. Kim SJ, Park YJ, Yang S-S, Kim Y-W (2013) Open surgical decompression of celiacaxis compression by division of the median arcuate ligament. Journal of the Korean Surgical Society 85: 93-95.

16. Bochenek A, Reicher M (1990). Human anatomy. Book for medical students and doctors. PZWL, Warsaw.

17. Jimenez JC, Harlander-Locke M, Dutson EP (20/2) Open and laparoscopic treatment of median arcuate ligament syndrome. J Vasc Surg; 56: 869-873.
18. Tulloch AW, Jimenez JC, Lawrence PF et al (2010) Laparoscopic versus open celiac ganglionectomy in patients with median arcuate ligament syndrome. J Vasc Surg 52: 1283-1289.

19. Dsaid SM, Zarroug AE, Gloviczki P, Shields RC (2010) Pediatric median arcuate ligament syndrome: first report of familial pattern and transperitoneal laparoscopic release. J Pediatr Surg; 45: EI7-E20.

20. Okten RS, Kucukay F, Tola M, Bostanci B, Cumhur T (2012) Is celiac artery compression syndrome genetically inherited? A case series from a family and review of the literature. Eur J Radiol; 81: 1089-1093.

21. Palmer OP, Tedesco M, Casey K, Lee JT, Poultsides GA (20I2) Hybrid treatment of celiac artery compression (median arcuate ligament) syndrome. Dig Dis Sci; 57: I782-I 785. 